\title{
The infrared Doppler (IRD) instrument for the Subaru telescope: instrument description and commissioning results
}

\section{Takayuki Kotani, Motohide Tamura, Jun Nishikawa, Akitoshi Ueda, Masayuki Kuzuhara, et al.}

Takayuki Kotani, Motohide Tamura, Jun Nishikawa, Akitoshi Ueda, Masayuki Kuzuhara, Masashi Omiya, Jun Hashimoto, Masato Ishizuka, Teruyuki Hirano, Hiroshi Suto, Takashi Kurokawa, Tsukasa Kokubo, Takahiro Mori, Yosuke Tanaka, Ken Kashiwagi, Mihoko Konishi, Tomoyuki Kudo, Bun'ei Sato, Shane Jacobson, Klaus W. Hodapp, Donald B. Hall, Wako Aoki, Tomonori Usuda, Shogo Nishiyama, Tadashi Nakajima, Yuji Ikeda, Tomoyasu Yamamuro, Jun-Ichi Morino, Haruka Baba, Ko Hosokawa, Hiroyuki Ishikawa, Norio Narita, Eiichiro Kokubo, Yutaka Hayano, Hideyuki Izumiura, Eiji Kambe, Nobuhiko Kusakabe, Jungmi Kwon, Masahiro Ikoma, Yasunori Hori, Hidenori Genda, Akihiko Fukui, Yuka Fujii, Hajime Kawahara, Guyon Olivier, Nemanja Jovanovic, Hiroki Harakawa, Masahiko Hayashi, Masahide Hidai, Masahiro Machida, Taro Matsuo, Tetsuya Nagata, Masahiro Ogihara, Hideki Takami, Naruhisa Takato, Hiroshi Terada, Daehyeon Oh, "The infrared Doppler (IRD) instrument for the Subaru telescope: instrument description and commissioning results," Proc. SPIE 10702, Ground-based and Airborne Instrumentation for Astronomy VII, 1070211 (13 July 2018); doi: 10.1117/12.2311836 


\title{
The infrared Doppler (IRD) instrument for the Subaru telescope: instrument description and commissioning results
}

Takayuki Kotani*a,b,c , Motohide Tamura ${ }^{a, b, d}$, Jun Nishikawa ${ }^{a, b, c}$, Akitoshi Ueda ${ }^{b}$, Masayuki Kuzuhara $^{\mathrm{a}, \mathrm{b}}$, Masashi Omiya ${ }^{\mathrm{a}, \mathrm{b}}$, Jun Hashimoto ${ }^{\mathrm{a}, \mathrm{b}, \mathrm{c}}$, Masato Ishizuka ${ }^{\mathrm{d}}$, Teruyuki Hirano ${ }^{\mathrm{e}}$, Hiroshi Suto $^{\mathrm{a}, \mathrm{b}}$, Takashi Kurokawa ${ }^{\mathrm{a}, \mathrm{f}}$, Tsukasa Kokubo ${ }^{\mathrm{f}}$, Takahiro Mori ${ }^{\mathrm{f}}$, Yosuke Tanaka ${ }^{\mathrm{f}}$, Ken Kashiwagi ${ }^{\mathrm{g}}$, Mihoko Konishia,b, Tomoyuki Kudo ${ }^{\mathrm{h}}$, Bun'ei Sato ${ }^{\mathrm{e}}$, Shane Jacobson', Klaus W. Hodapp ${ }^{\mathrm{i}}$, Donald B. Hall $^{i}$, Wako Aoki ${ }^{\text {bcc }}$, Tomonori Usuda ${ }^{b, c}$, Shogo Nishiyama ${ }^{j}$, Tadashi Nakajima ${ }^{a, b}$, Yuji Ikeda ${ }^{k}$, Tomoyasu Yamamuro ${ }^{1}$, Jun-Ichi Morino ${ }^{\mathrm{b}}$, Haruka Baba ${ }^{\mathrm{c}}$, Ko Hosokawa ${ }^{\mathrm{c}}$, Hiroyuki Tako Ishikawa ${ }^{\mathrm{c}}$, Norio Narita ${ }^{\mathrm{d}}$, Eiichiro Kokubo ${ }^{\mathrm{b}}$, Yutaka Hayano ${ }^{\mathrm{b}}$, Hideyuki Izumiura ${ }^{\mathrm{n}}$, Eiji Kambe ${ }^{\mathrm{h}}$, Nobuhiko Kusakabe $^{\mathrm{a}, \mathrm{b}}$, Jungmi Kwon ${ }^{\mathrm{m}}$, Masahiro Ikoma ${ }^{\mathrm{d}}$,Yasunori Hori ${ }^{\mathrm{a}, \mathrm{b}}$, Hidenori Genda ${ }^{\mathrm{e}}$, Akihiko Fukui ${ }^{\mathrm{n}}$, Yuka Fujii ${ }^{\mathrm{e}}$, Hajime Kawahara ${ }^{\mathrm{d}}$, Olivier Guyon ${ }^{\mathrm{h}}$, Nemanja Jovanovic ${ }^{\mathrm{q}}$, Hiroki Harakawa ${ }^{\mathrm{a}, \mathrm{b}}$, Masahiko Hayashi $^{\mathrm{b}}$, Masahide Hidai ${ }^{\mathrm{p}}$, Masahiro Machida ${ }^{\mathrm{q}}$, Taro Matsuo ${ }^{\mathrm{r}}$, Tetsuya Nagatas, Masahiro Ogihara $^{\mathrm{b}}$, Hideki Takami ${ }^{\mathrm{b}}$, Naruhisa Takato ${ }^{\mathrm{h}}$, Hiroshi Terada ${ }^{\mathrm{h}}$, Daehyeon $\mathrm{Oh}^{\mathrm{t}}$, IRD team

${ }^{a}$ Astrobiology center, 2-21-1 Osawa, Mitaka, 181-8588, Japan

bNational Astronomical Observatory of Japan, 2-21-1 Osawa, Mitaka, 181-8588, Japan

'GUAS, 2-21-1, Osawa, Mitaka, Tokyo, 181-8588, Japan

dThe University of Tokyo, Hongo, Tokyo 113-0033, Japan

'Tokyo Institute of technology, Meguro, Tokyo 152-8550, Japan

f Tokyo University of Agriculture and Technology, Koganei, Tokyo 184-8588, Japan

gNational Institute of Advanced Industrial Science and Technology, 1-1-1 Umezono, Tsukuba, Ibaraki 305-8560 Japan

hSubaru Telescope, 650 North A'ohoku Place, Hilo, HI 96720, USA

institute for Astronomy, University of Hawaii, 640 North A'ohoku Place, Hilo, HI 96720, USA

${ }^{\mathrm{j} M i y a g i}$ University of Education, 149, Aramaki-aza-Aoba,Aobaku, Sendai, 980-0845, Japan

kPhotocoding, 460-102, Iwakura Nakamachi, Sakyo-ku, Kyoto 606-0025, Japan

'Opt-craft, 101, 3-16-8, Higashi-Hashimoto, Midori-ku, Sagamiha, 252-0144, Japan

mISAS/JAXA, 3-1-1Yoshino-dai, Sagamihara,Kanagawa 252-5210, Japan

${ }^{\mathrm{n}} \mathrm{OAO}$, National Astronomical Observatory, Okayama 719-0232, Japan

${ }^{\circ}$ California Institute of Technology, 1200 east California boulevard, Pasadena, California 91125

pTokai university, 4-1-1 Kitakaname, Hiratsuka-shi, Kanagawa, 259-1292 Japan

qKyusyu University, Fukuoka, 812-8581, Japan

rOsaka University, 1-1 Yamadaoka, Suita, Osaka 565-0871 Japan

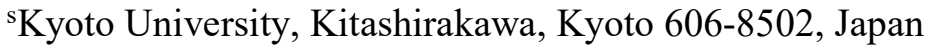

'NMSC Korea, 64-18, Guam-gil, Gwanghyewon-myeon, Jincheon-gun, Chungcheongbuk-do 27803, Korea

\begin{abstract}
The Infrared Doppler (IRD) instrument is a fiber-fed high-resolution NIR spectrometer for the Subaru telescope covering the Y,J,H-bands simultaneously with a maximum spectral resolution of 70,000. The main purpose of IRD is a search for Earth-mass planets around nearby M-dwarfs by precise radial velocity measurements, as well as a spectroscopic characterization of exoplanet atmospheres. We report the current status of the instrument, which is undergoing commissioning at the Subaru Telescope, and the first light observation successfully done in August 2017. The general
\end{abstract}

Ground-based and Airborne Instrumentation for Astronomy VII, edited by Christopher J. Evans, Luc Simard, Hideki Takami, Proc. of SPIE Vol. 10702, 1070211 - (c) 2018 SPIE · CCC code: 0277-786X/18/\$18 · doi: 10.1117/12.2311836 
description of the instrument will be given including spectrometer optics, fiber injection system, cryogenic system, scrambler, and laser frequency comb. A large strategic survey mainly focused on late-type M-dwarfs is planned to start from 2019.

\section{GENERAL DESCRIPTION OF THE INSTRUMENT}

The Infrared Doppler (IRD) instrument is a fiber-fed high-resolution near-infrared (NIR) spectrometer for the Subaru telescope covering the Y-, J-, and H-bands simultaneously with a maximum spectral resolution of 70,000. In this section, we give a general description and the expected performance of the instrument, which is now undergoing commissioning at the Subaru telescope. Commissioning at the summit started in March 2017 and first light observations were successfully carried out in August 2017. The laser frequency comb is fully available combined with IRD since January 2018. This section includes an overview of the spectrometer optics, fiber injection system, cryogenic system, scrambler, and laser frequency comb. The general schematic view of the instrument is shown in Figure 1 and the important characteristics of IRD are summarized in Table 1. The spectrometer is designed to cover wavelengths from 970-1750 nm with a 70,000 maximum spectral resolution. The instrument consists of 4 main components: a fiber injection module mounted on the adaptive optics bench at the Nasmyth platform, mode scramblers to reduce a modal noise, a spectrometer enclosed in a vacuum chamber in the Coudé room, and the laser frequency comb system as a wavelength reference.

Table 1. Specifications of IRD instruments

\begin{tabular}{|l|l|}
\hline Wavelength coverage & $970-1750 \mathrm{~nm}$ \\
\hline Spectral resolution & $70,000 \mathrm{max}$ (Multi-mode fiber), 100,000 (Single-mode fiber) \\
\hline Detector and controller & $2 \times \mathrm{HAWAII}$ RG and Sidecar-ASIC \\
\hline Fiber diameter and FOV & $\begin{array}{l}60 \mu \mathrm{m}, 0.48 \text { arcsec (MMF: OFS F8950), 7.5 } \mathrm{mm} \text { MFD@1550nm (SMF: OFS } \\
\text { BF05635-02) }\end{array}$ \\
\hline Wavelength reference & Laser frequency comb, ThAr lamp \\
\hline Throughput & $2.3 \%$ at $\sim 1000 \mathrm{~nm}, 3.9 \%$ at $\sim 1520 \mathrm{~nm}$ (Blaze peak) \\
\hline Dispersive optics & High blaze angle Echelle grating (78.98-degree blaze angle) + VPH cross-disperser \\
\hline Temperature & $\sim 79 \mathrm{~K}$ (detectors), 61K (camera lens), 180K (optical bench and other optics) \\
\hline Cryocooler & $2 \times$ pulse-tube crycoolers (1 for Detectors, camera lens and 1 for the optical bench) \\
\hline Adaptive optics & 188 -element AO188 or Extreme-AO (Subaru Coronagraphic Extreme AO) \\
\hline
\end{tabular}

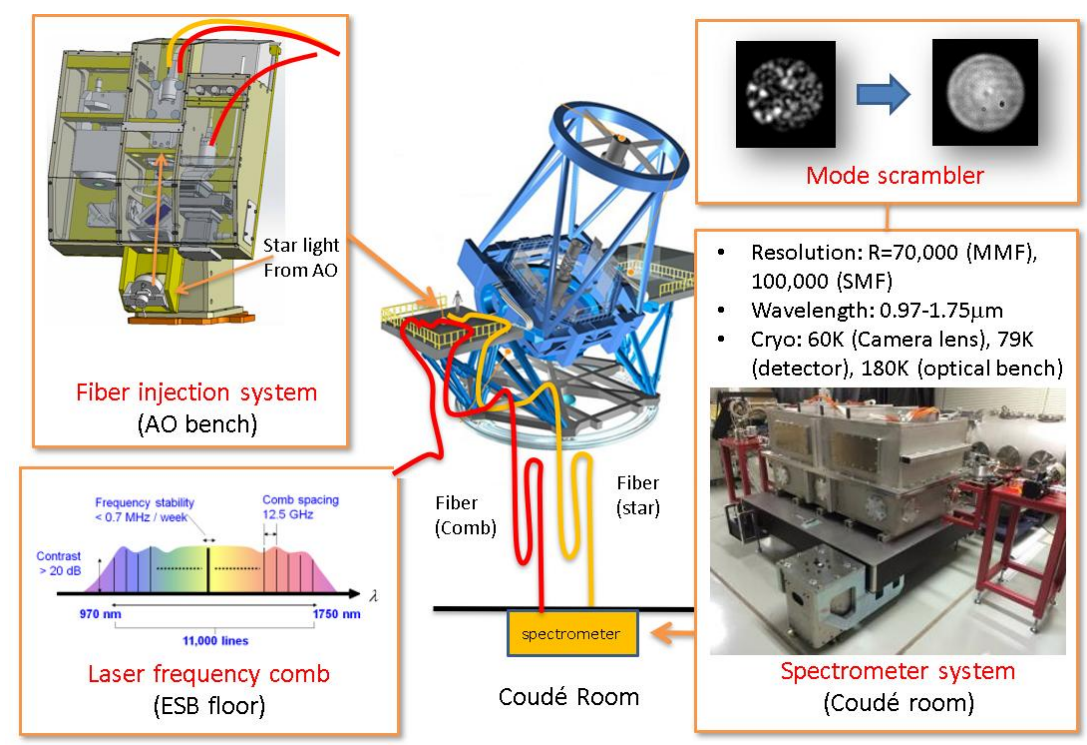

Figure 1. Schematic view of the IRD instruments. 


\section{FIBER INJECTION MODULE}

A fiber injection module (FIM) is mounted on the optical bench of the adaptive optics AO188 at the infrared Nasmyth platform to inject AO-corrected star light and a laser frequency comb into two different fibers (Figure 2). The FIM was designed and fabricated by Opt-craft The FIM is mounted on a motorized translation stage on the AO bench so that the instrument exchange can be done quickly for future que-mode operation. The F ratio of the input beams is converted from $\mathrm{F} / 13.9$ to $\mathrm{F} / 3.33$ in order to mitigate focal ratio degradation (FRD) of a multi-mode fiber (MMF). At the focus of the F-conversion optics, a fused silica v-roove fiber array which contains $2 \times 60 \mu \mathrm{m}$ core MMFs (OFS F8950 from Furukawa Electric Co., Ltd) separated by $2.44 \mathrm{~mm}$ (18.9 arcsec). A SMF (OFS BF05635-02) is located in the middle of the two MMFs. A star-light and a laser comb light can be injected into two different fibers respectively via telescope pointing and moving a pick-off mirror to feed a laser comb light from a laser comb fiber. The Field-Of-View (FOV) of MMF is 0.48 arcsec and a typical injection efficiency with AO188 is around $60 \%$ for a normal seeing condition. Light of a laser frequency comb emerged from a MMF, which is connected to the laser frequency comb system installed near the oude room, is sent to a F-conversion lens system by a motorized pick-off mirror around the AO focus. The reflected light by a pin-hole mirror is sent to the monitoring camera for real-time star position measurements. The camera consists of an imaging lens system (F/5, 30 arcsec effective FOV, 0.065 arcsec/pixel) and the NIR sensitive CCD (pixel scale $=13 \mu \mathrm{m}$, Bitran BK50 NIR) for precise star light coupling into a fiber. A piezo-driven tip-tilt mirror can correct a slow drift of image position arising from residual tip-tilt errors of AO188 and atmospheric dispersion effect to realize less than $10 \mathrm{mas}$ rms drift during an exposure. The star-light position can be monitor in real-time thanks to the pin-hole mirror mounted at the $\mathrm{AO}$ focus and reflected light from the pin-hole mirror and the pick-off glass plate by Fresnel reflection is sent to a monitor camera. The light from 950 to $1000 \mathrm{~nm}$ can be used to monitor the star light position.

A star-light injection to a SMF is an attractive option because a SMF has no modal noise above the cut-off wavelength. On the other hand, it is difficulty to realize high coupling efficiency to a SMF if wavefront error (WFE) of the input beam is large. Therefore, a SMF injection with AO188 would be limited to only a bright star. A combination of IRD and Subaru Coronagraphic Extreme AO (SCExAO) will enable us to achieve very high efficiency start light injection to a SMF and it will be realized in 2018.

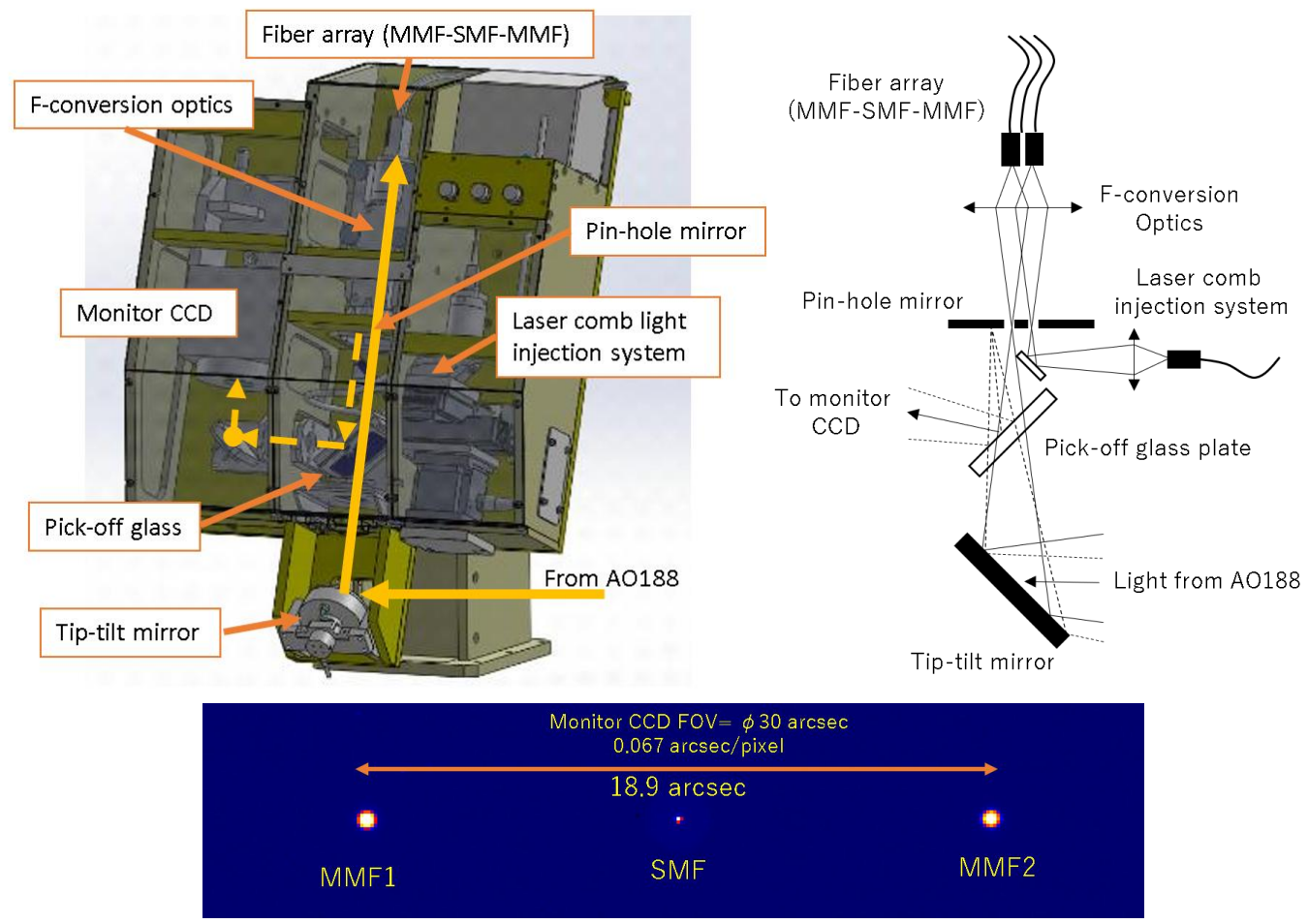

Figure 2. Left top: 3D model of the fiber injection module. Right top: The schematic view of the optical system of FIM. Bottom: Monitoring CCD view of $2 \times \mathrm{MMF}$ and $1 \mathrm{SMF}$ 


\section{SPECTROMETER}

The spectrometer optical design and fabrication of the camera lens was done by Photocoding. The spectrometer employs a new high-blazed echelle grating and a VPH cross-disperser to achieve a spectral resolution of 70,000 at maximum, covering the Y-, J-, H-bands $(970-1750 \mathrm{~nm})$ simultaneously with two $2.5 \mu \mathrm{m}$ cutoff Teledyne H2RG detectors. Two fibers can be used simultaneously to record a spectrum of a star and a wavelength reference source, a laser frequency comb (LFC). There are no moving parts in the spectrometer optics and the spectrometer is enclosed in a vacuum chamber located in the Coudé room of the Subaru Telescope for good temperature stability. Figure 3 shows the raytracing model of the spectrometer optics. In Figure 4, the spectral resolution and total efficiency of the system is shown.

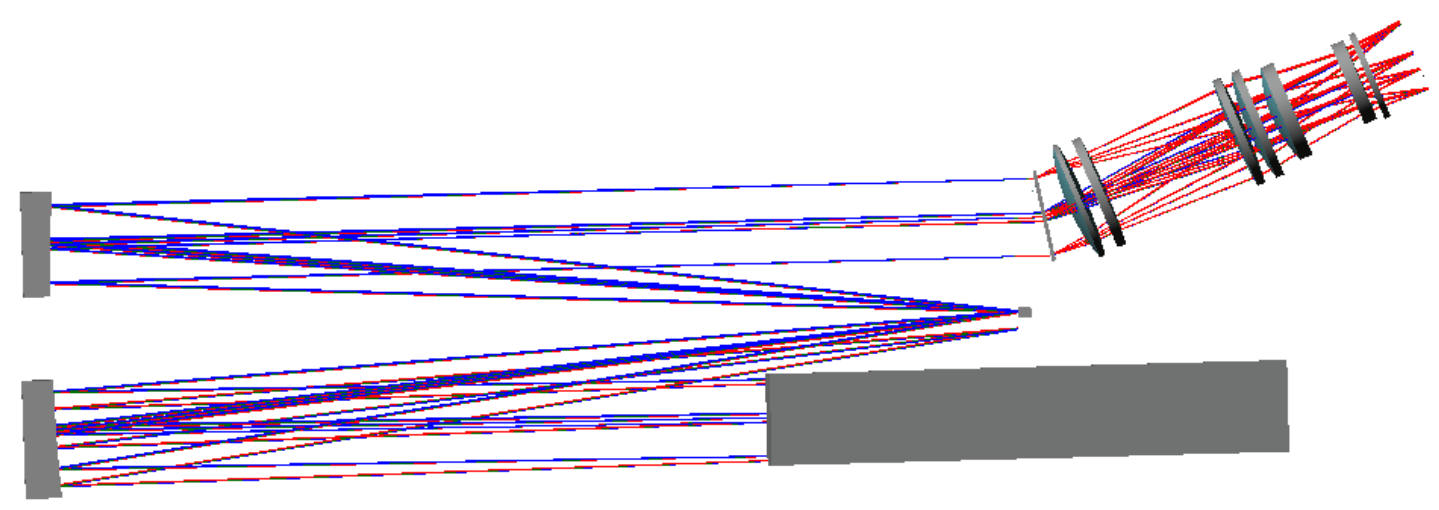

Figure 3. Ray-trace model of the spectrometer optics. Note that F-conversion optics is not included in this figure.

1) Fiber-feed system: 2 MMFs (OFS F8950) and 2 SMFs (OFS BF05635-02) are introduced into the chamber by using a vacuum feed-through connector (SEDI-ATI fibres optiques). One fiber is used for a star light and another one is for the laser comb light. In the chamber, the fibers are aligned linearly with $0.179 \mathrm{~mm}$ spacing and mounted on a fused-silica vgroove array (HATAKEN Co., LTD). AR coating from 970-1750 nm is applied to the output surface of the fiber array. We can select any two fibers MMF-MMF/MMF-SMF/SMF-SMF simultaneously. The beams emerged from the fibers are imaged on a slit after F-conversion (3.33 to 12.65$)$. The slit has $0.129 \mathrm{~mm}$ width $\times 1.053 \mathrm{~mm} \mathrm{long}(0$ ".27 $\times 2$ ". 2$)$ and the slit width corresponds to $64 \%$ of the size of the re-imaged $60 \mu \mathrm{m}$ core fiber. This will increase the spectral resolution at the cost of $30 \%$ throughput loss. This fiber-feed system was designed and fabricated by Opt-craft.

2) Collimator and Gratings: After the slit, the beams are sent to a collimator-grating module. The collimator consists of two off-axis parabola mirrors $(\mathrm{f}=741.1 \mathrm{~mm})$ with slightly shifted each other in order to reduce aberrations. After collimation, the beams are dispersed by a newly developed Echelle grating (Richardson grating laboratory). The grating has 13.3 grooves $/ \mathrm{mm}$ and its blaze angle is 78.98 degrees. The grating substrate is Zerodur and the replicated grating surface is gold coated. The dimension is $410 \mathrm{~L} \times 90 \mathrm{~W} \times 74 \mathrm{H} \mathrm{mm}$. The dispersed beams are re-collimated by the collimator mirror system to make a re-imaged pupil at a Volume-Phase Holographic (VPH) grating (cross disperser, 278 line $/ \mathrm{mm}$, Angle of Incidence $=10.9$ degrees, Angle of Diffraction $=-10.9$ degrees, $102 \times 79 \times 6$ mm, Kaiser optical system inc.).

3) Camera and detector assembly: The spectrum is imaged onto $2 \times$ HAWAII2-RG detector arrays through the camera optics (focal length $=249 \mathrm{~mm}$ ). Thanks to a large format array, wide spectral coverage $(970-1750 \mathrm{~nm})$ can be covered simultaneously. High atmospheric absorption band at the edge of the the H- and J-band (1370-1420nm) falls onto the gap between the two detectors, therefore the loss of the spectrum due to the gap is negligible. A thermal cut and order-sorter filter, coated to one 5-mm thick Infrasil glass plate fabricated by Optical Coatings Japan, is placed just in front of the detector array. The focus of the detectors was precisely adjusted by putting shims between the backend of the camera lens module and the detectors assembly. VPH, camera lens, and the detectors module are all connected together by screws and makes it to one module with no moving parts for good stability. This module is supported by $3 \times 1 \mathrm{~mm}$ thick 
super-invar plates in order for thermal isolation from the bench and for absorbing differential deformation due to the different temperature condition between the module $(\sim 60 \mathrm{~K})$ and the optical bench $(\sim 180 \mathrm{~K})$.

4) Ultra-low CTE ceramic optics: the optical bench, off-axis parabolas and their holders, the flat mirror, Echelle grating holder, the slit-F-conversion unit holder, a baseplate for the camera-lens and detector module, are made of ultra-low thermal expansion ceramic (Figure 5, cordierite from Kyocera. Coorp., $\mathrm{CTE}<\left|0.05 \times 10^{-6}\right|$ at room temperature). The poreless ceramic CO720 was used for off-axis parabolas, a flat mirror and CO220 was used for the optical bench and a slit holder, and a baseplate. The characteristics of CO220 and CO720 are almost same, except that CO720 has much less pore or void in its surface, hence it has better surface finish after polishing. The surface roughness of the off-axis parabolas and the flat mirror is less than $3 \mathrm{~nm}$ Ra and the surface figure error is $<\lambda / 4 \mathrm{PV}$ at $\lambda=633 \mathrm{~nm}$, which means that there is no problem for near-infrared usage. Mirror surface was gold coated and reflectance is over 98\% from 970$1750 \mathrm{~nm}$. The optical bench has $1300 \mathrm{~L} \times 600 \mathrm{~W} \times 25 \mathrm{Hmm}$ dimension and it is reinforced by $100 \mathrm{~mm}$ height rib structures. These optical components are precisely machined and positioned by using a 3D coordinate-measuring machine. The same material used for the optics and the optical bench enables the optical alignment remain stable after cooling process. Figure 8 shows the ceramic optical bench, the mirrors and the slit holder.
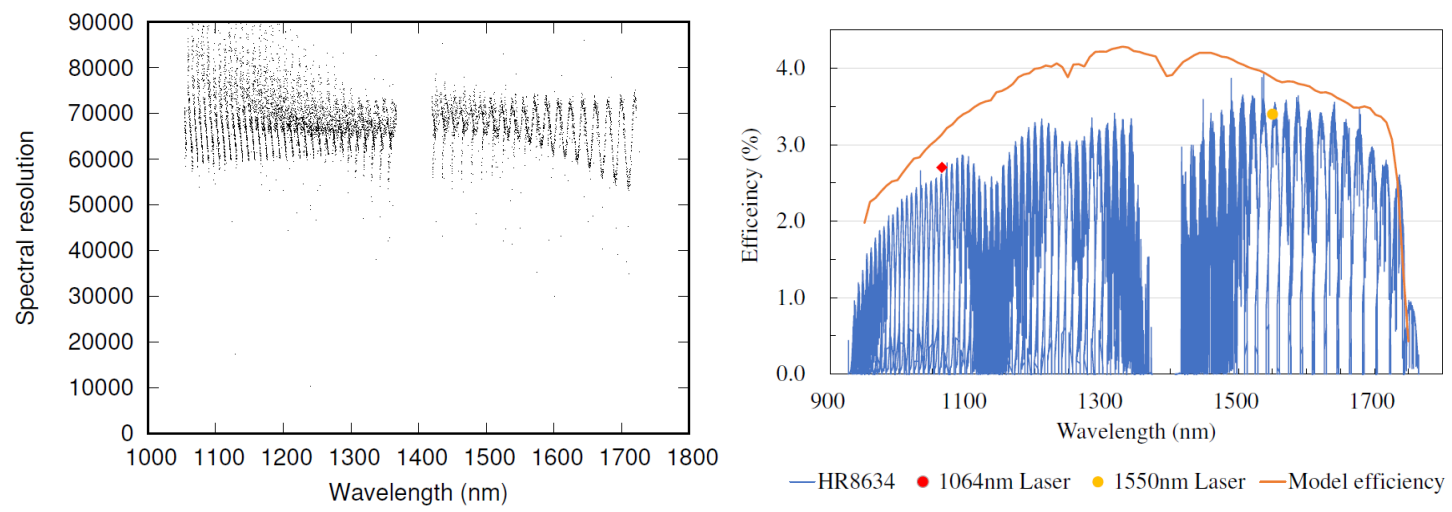

Figure 4. Left: Spectral resolution of the spectrometer. Right: Total efficiency including the telescope, a fiber, spectrometer, detector QE, etc. measured with a star.
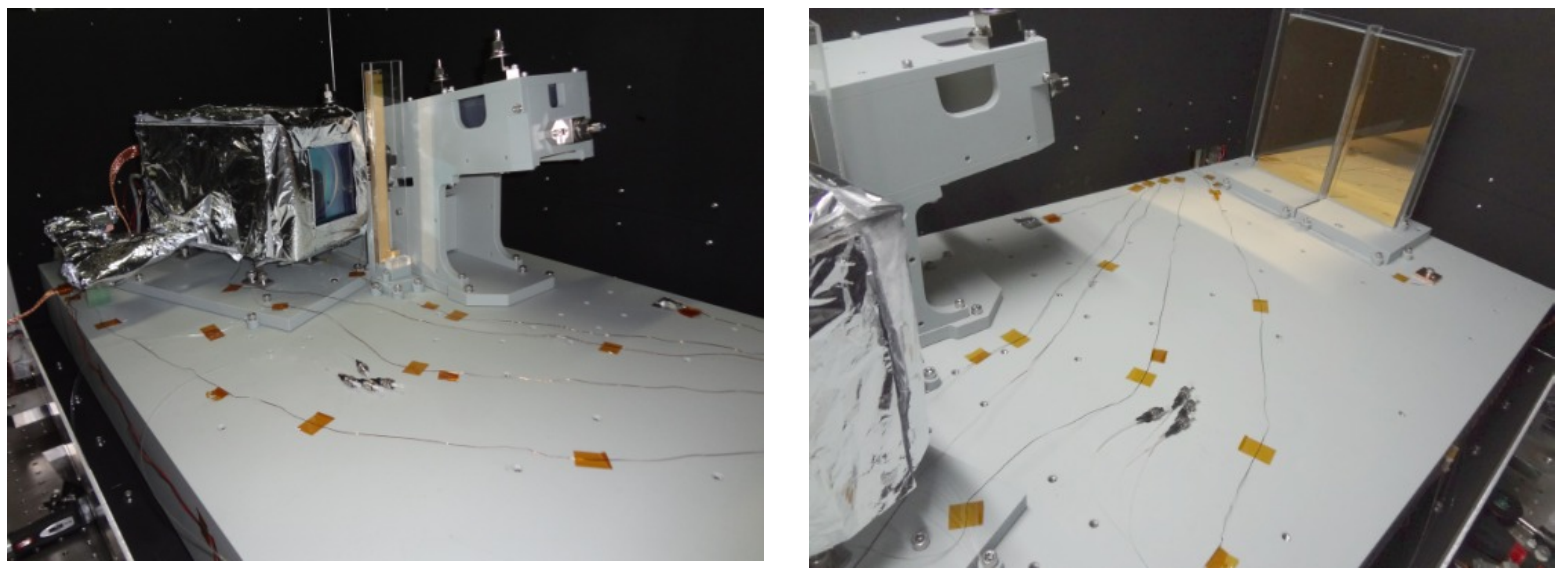

Figure 5. Picture of the spectrometer. The light green components are made of ceramic (Kyocera cordielite). 


\section{VACUUM AND CRYOGENIC SYSTEM}

The spectrometer and detector systems are enclosed in a vacuum chamber designed and fabricated by VIC International Co., Ltd. We employ two low-vibration pulse tube cryocoolers (PDC08 and PC150 from Ulvac cryogenics inc.) for cooling the camera lens-detector assembly and other optics respectively (Figures 6 and 7). The camera lens-detector assembly is connected to the cold-head via oxygen-free copper (OFC) thermal strap and plate for efficient cooling. All optical system including the optical bench, Echelle grating, mirrors, camera lens system, etc., are enclosed by aluminum enclosure, inner side is black painted with CA8211 and CA7233 (PPG aerospace) to which the thermal strap from the other cold-head is connected, in order to realize a uniform cooling by radiative cooling. The aluminum enclosure is suspended by three $5 \mathrm{~mm}$ thick, $40 \times 420 \mathrm{~mm}$, G-10 glass epoxy plate (Nikolyte NL-EG-TR from Nikko Kasei CO. LTD) for thermal insulation from the vacuum chamber. The enclosure and camera lens-detector assembly, thermal straps are covered with 10-layer Multi-Layer Insulation (MLI, COOLCAT 2NW from RUAG space) to block thermal radiation from the environment. As for the thermal strap, two types of straps were fabricated; laminated $16 \mu \mathrm{m}$ OFC sheet (Figure 8, Hitachi Metals Neomaterial, Ltd), consisting of more than 300 stacked sheets pressed into copper blocks at both ends; a braided copper wire, welded to a copper block. Both thermal straps are flexible and has a good thermal conductivity. At a connection part of the thermal strap, we put an indium sheet $(0.1 \mathrm{~mm}$ thick) for a good thermal conductivity between two metal blocks. In addition, the coldheads are electrically isolated from the compressors and the motor units of the cryocoolers to block an electric noise to the detectors. To realize both electric isolation and good thermal conductivity, several CVD diamond plates (TM100,10x10mm, 0.5mm thick from Element Six Technologies) are inserted between the coldheads and the copper thermal path.

The temperatures of the camera lens and the detector system are cooled down to $\sim 61 \mathrm{~K}$ and $79 \mathrm{~K}$ respectively; the other parts of the optical system are kept around $180 \mathrm{~K}$ by radiative cooling. Temperature sensor is Lakeshore DT-670-CU-1.4 and temperature control is realized by Kapton insulated heaters (Minco Products, Inc. and OMEGA Engineering, INC.) and Lakeshore 336 controllers. Heaters are attached to the following places via Stycast 2850FTJ and CATALYST 9M; 2 $\times$ heaters to $2 \times$ cold-head, 1 to the camera lens barrel, 6 to aluminum enclosure panel. Figure 8 shows the temperature control unit for one of the coldheads (PDC08).

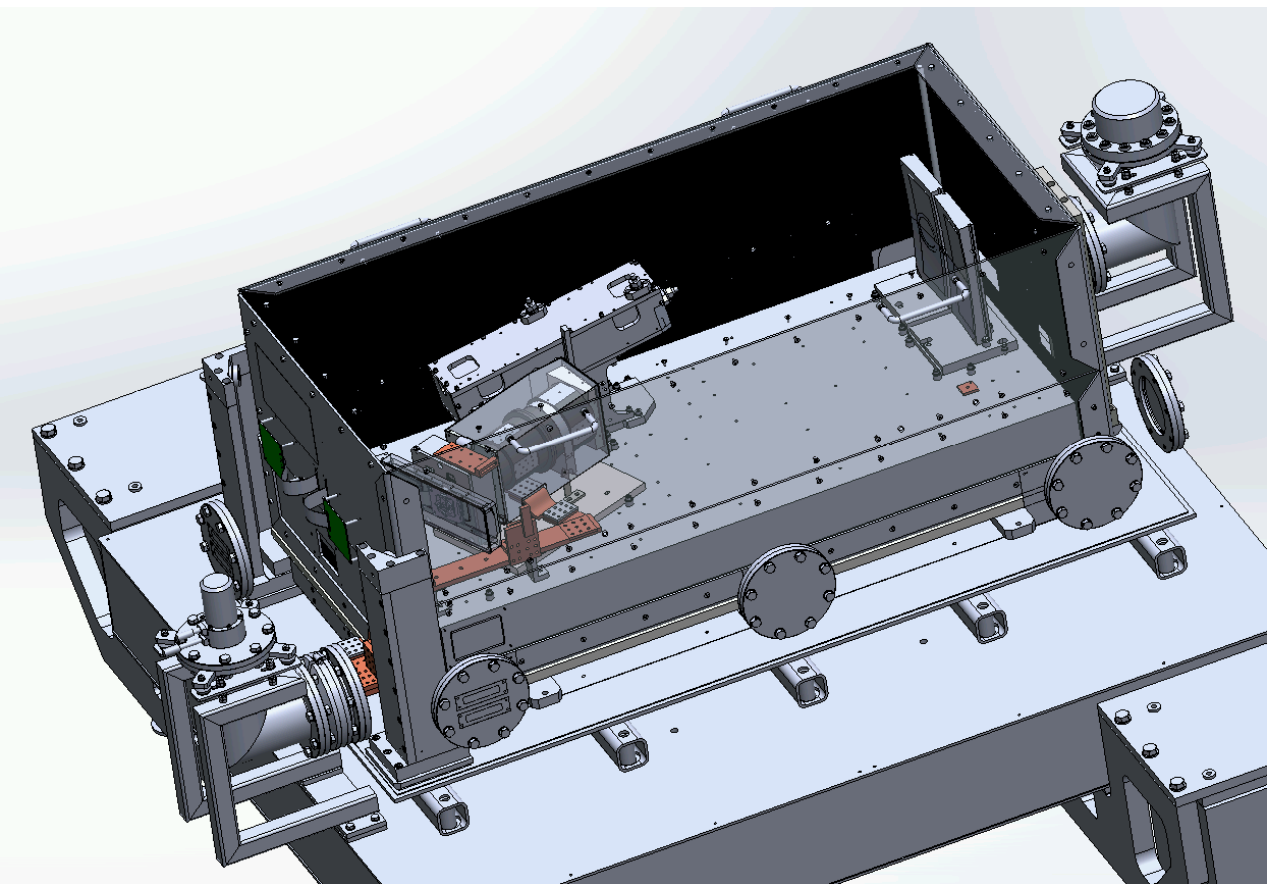

Figure 6. Transparent view of the vacuum chamber and internal components. 


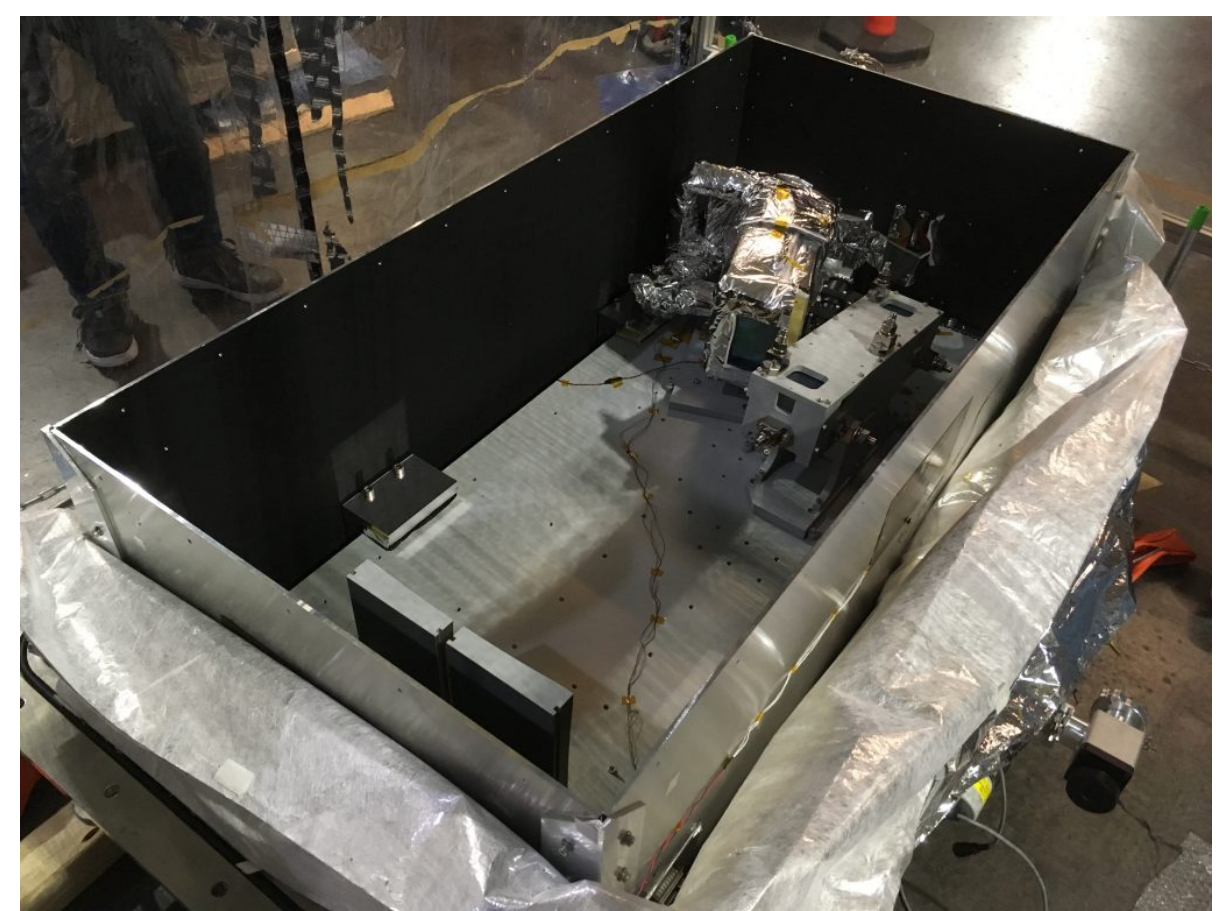

Figure 7. Spectrometer optics and optical bench enclosed by the radiation shield and aluminum enclosure
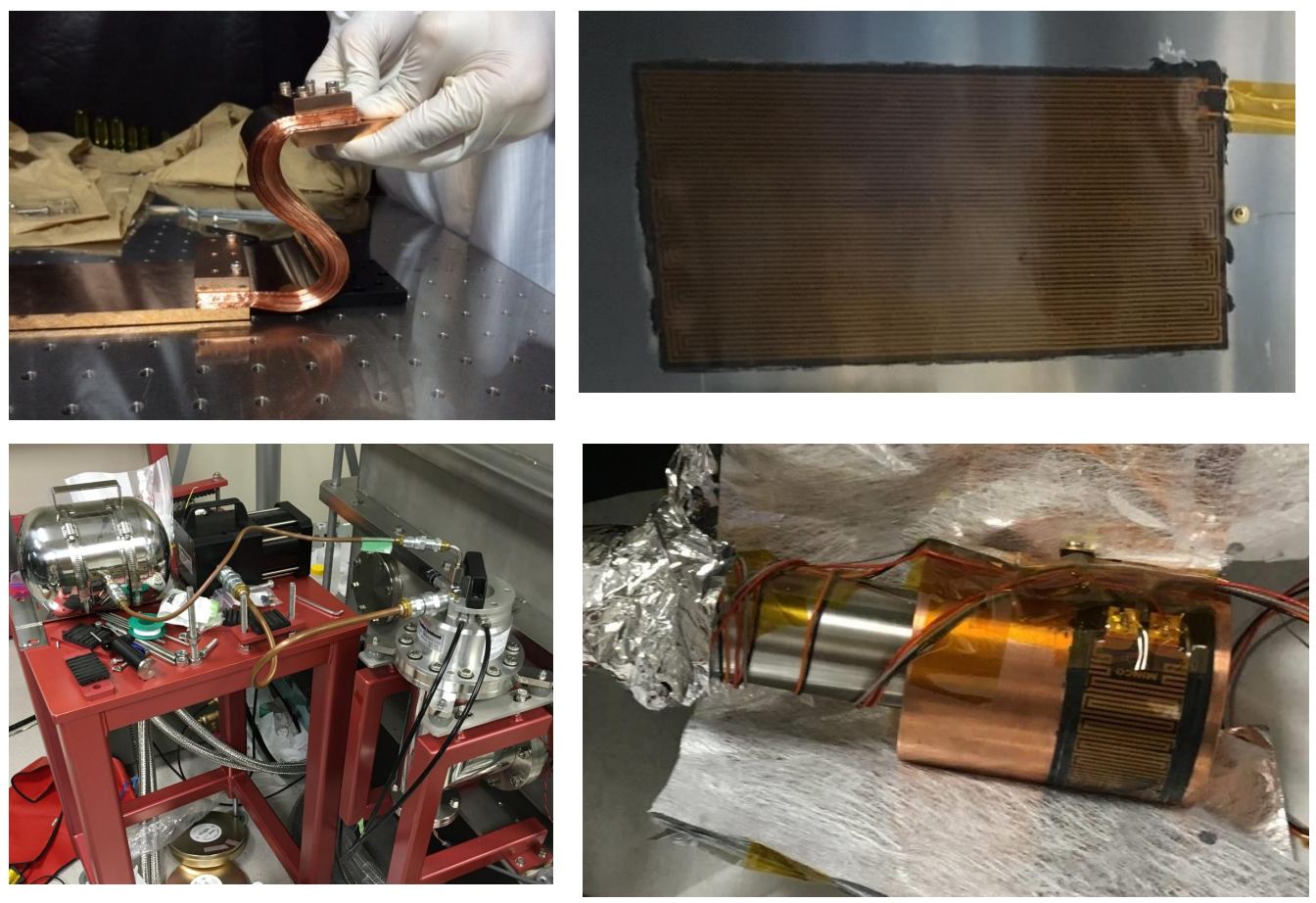

Figure 8. Left top: Flexible copper thermal strap. Right top: Kapton heater. Left bottom: One of the pulse-tube cryocooler. Right bottom: Temperature control unit for the coldhead 


\section{MODE SCRAMBLER}

Modal noise is one of the major error sources in RV measurements, therefore a performance of a mode scrambler is critical especially in the NIR A scrambling method should be optimized for highly coherent light like the laser comb and partially coherent star light respectively, because scrambling of coherent light is more difficult. Figure 9 shows the schematic view of IRD's scrambler system. From our detailed study of various scramblers ${ }^{1}$, we selected following scramblers: 1) Star scramble: 200-meter MMF + bending scrambler + 200-meter MMF + bending scrambler. 200-meter fiber is rounded around a $16 \mathrm{~cm}$ diameter bobbin. The bending scrambler is a 16-meter, loosely rounded fiber of which one side of the loop is fixed to a seesaw like shaker instrument (NX-11-20, Nisshin Rika) and the other side of the fiber loop is attached to the vertical stand (Figure10). The seesaw shaker moves the fiber loop at $\sim 1 \mathrm{~Hz}$. For the laser comb, more powerful scramblers are used; LFC light passes a combination of rotating holographic diffusers (Edmund optics $2 \times$ $\# 55-848$ and $1 \times \# 55-849$ ) to reduce a speckle pattern emerged from a MMF; $1 \mathrm{~km}$ fiber as a static scrambler; all-fiber twisting scrambler using $200 \mu \mathrm{m}$ core fiber (Fiberware S200/280 IPRI, GiGa Concept Inc.), and $2 \times$ bending scramblers which are same as the star scrambler. In addition to these scramblers, we also use a polarization scrambler because the LFC light is highly polarized and a spectrometer response is very sensitive to variations of the polarization state of the input light. The polarization scrambler consists of a collimator and focusing lens system, achromatic half and quarterwave plate (Thorlabs SAHWP05M-1700 and SAQWP05M-170), rotating with a frequency of 1.92 and $0.97 \mathrm{~Hz}$ respectively, placed in the collimated beam. This "depolarizer" ensures almost random polarization state in case of a long exposure observation (typically longer than a few minutes).

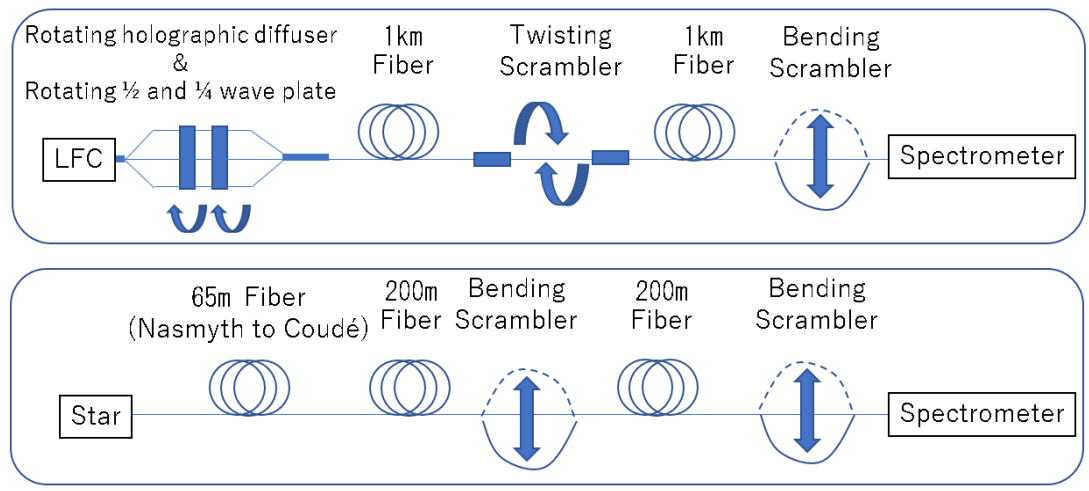

Figure 9. Mode scramblers employed for IRD. Optimized scramblers for LFC and star light respectively.

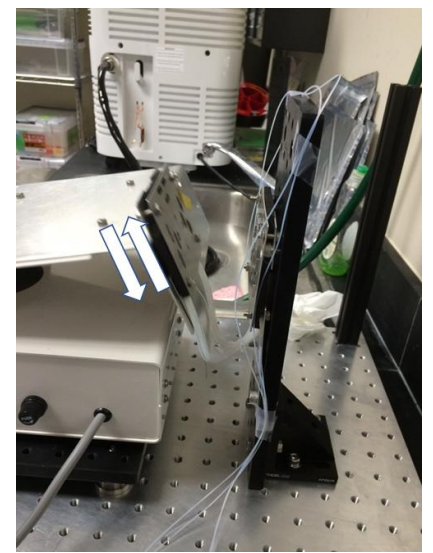

Figure 10. Dynamic bending scrambler 


\section{LASER FREQUENCY COMB}

A newly developed laser frequency comb (LFC) covers 1050-1750 nm, almost the whole Y-, J-, H-bands, with a 12.5 $\mathrm{GHz}$ frequency interval ${ }^{2}$. This provides very precise wavelength standard for IRD. Based on a pulse shaping technology using a frequency-stabilized laser, the broadband LFC with a wide mode spacing was directly generated using highly nonlinear fibers. Though the original comb spectrum had peaks and troughs, it was flattened to within about $5 \mathrm{~dB}$ across the range using a spectrum shaper consisting of a fiber Bragg grating, fixed optical filters, and a liquid crystal based programmable filter. Figure 11 depicts two-dimensional echelle spectrum of the LFC and a star.
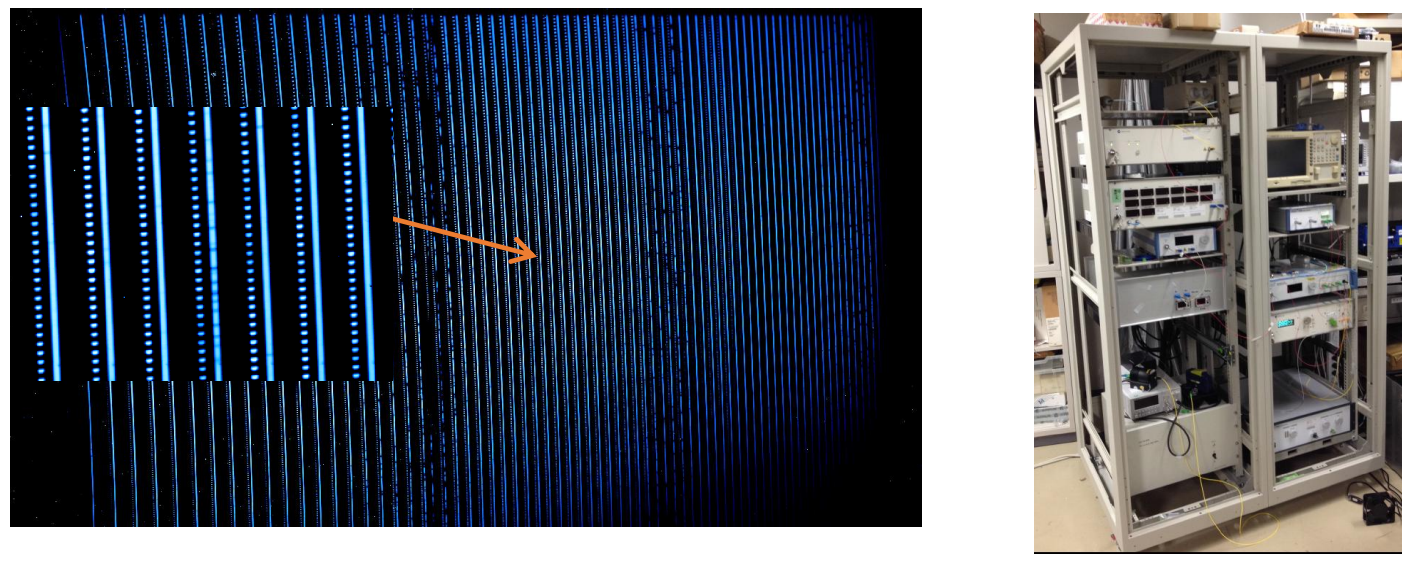

Figure 11. Left: Two-dimensional Echelle spectrum of LFC and star light. Right: Laser comb system mounted in the 19-inch racks

\section{ERROR BUDGET ANALYSIS AND ON-SKY RESULTS}

In order to predict IRD's RV stability, we performed an error budget analysis by splitting the LFC's light between two fibers. This enabled us to simulate relative RV measurements, which are similar to what we will actually obtain in an observation. Details are explained in Kuzuhara et al. ${ }^{3}$, but we briefly report the result in this paper.

Table 2 shows the summary of the error budget analysis. The data for this error budget analysis were collected for $\sim 2$ weeks and we will continue to obtain data to study more long-term stability. The results of the error budget analysis are shown in Table 2 and the expected total instrumental error is $1.3 \sim 1.9 \mathrm{~m} / \mathrm{s}$. The RV error can be attributed to five main sources: 1) The wavelength stability of the LFC depends on the ambient temperature. The LFC system is enclosed in a temperature controlled room to ensure a good stability 2) Detector intra- and inter-pixel sensitivity variations introduce an apparent change of the Instrumental Profile (IP), when an absolute drift of the spectrum exists. Because the star and the LFC spectrum fall on different detector pixels and the pixel sensitivity varies across the chip, this will lead to a differential spectrum drift which cannot be corrected with the LFC. 3) and 4) Modal noise is one of the major error sources especially for a NIR spectrometer, and it is essentially an actual instability of IP caused by various reasons; telescope pointing variation, thermal deformation of the optics, temperature changes and mechanical deformation of an optical fiber, etc. The modal noise for the LFC and a star light should behave independently because the modal noise tends to be high in the case of the very high coherent light like LFC, in contrast to partially coherent star light. Therefore, we characterized the modal noises each for the LFC and the star light. 5) The spectrometer response, in terms of RV measurements, is very sensitive to the polarization state of the input light. Especially the LFC light is highly linearly polarized and it can cause $>10 \mathrm{~m} / \mathrm{s}$ drift at the worst case from our laboratory experiment. We employ a polarization scrambler to reduce the degree of polarization in a long-exposure case. 
Table 2. Summary of the error budget analysis.

\begin{tabular}{|l|l|l|}
\hline Error source & Error budget & \multicolumn{1}{|c|}{ Measurement method } \\
\hline Laser comb stability & $0.04 \mathrm{~m} / \mathrm{s} /$ week & Heterodyne interferometry \\
\hline $\begin{array}{l}\text { Detector inter- and intra- pixel sensitivity } \\
\text { variation }\end{array}$ & $0.96 \mathrm{~m} / \mathrm{s}$ & MMF-MMF \\
\hline Modal noise (laser comb) & $<0.7 \mathrm{~m} / \mathrm{s}$ & MMF-SMF \\
\hline Modal noise (star) & $0.5 \sim 1.2 \mathrm{~m} / \mathrm{s}$ & Laser beam centroid \\
\hline Polarization (laser comb) & $<0.77 \mathrm{~m} / \mathrm{s}$ & Polarized SMF beam \\
\hline Total instrumental noise & $1.3 \sim 1.9 \mathrm{~m} / \mathrm{s}$ & \\
\hline
\end{tabular}

\subsection{Preliminary on-sky results}

We have undertaken observations of RV standard stars, Tau Ceti (G8V), and the famous M-dwarf, Barnard's star (M4V) to demonstrate the instrument stability on-sky since August 2017. Note that we had to use a Thorium-Argon lamp (ThAr), a classical wavelength reference often used for the optical spectrometers in 2017, because of a malfunction of the LFC. The number of emission lines from the ThAr lamp is significantly smaller than that of the LFC and it certainly degrades the wavelength calibration. The LFC has been recovered in January 2018 and now it functions properly. Even with the ThAr lamp calibration, the RV error in the data of Tau Ceti and Barnard's star is $\sim 3.1$ and $2.5 \mathrm{~m} / \mathrm{s} \mathrm{rms}$ over a few nights observations, respectively. We will continue observations of RV standard stars to characterize a long-term stability of the instrument.

\section{SURVEY PLAN}

Our survey plan in the framework of the Subaru Strategic Program (SSP) focuses on a planet mainly around late Mdwarf. IRD is ideally suited to measure precise RVs of, especially late type M-dwarfs because: 1) late-type M-dwarfs are generally fainter than early type stars, therefore a large telescope aperture, i.e. 8-meter class telescope, is required for efficient observations. 2) late type M-dwarfs have many absorption lines in the H-band in addition to the Y-band, therefore simultaneous, $\mathrm{Y}$ to $\mathrm{H}$-band observation is important for better RV precision. IRD covers these important wavelengths. 3) those lines are less affected by stellar activity than optical lines. 4) Selection of "very quiet" star (less active stars) is particularly important for high precision RV measurements. It will lead to observe relatively faint stars because of selection effect. Large aperture telescope is again critical to observe such quite stars.

The goals of the survey are: (1) to discover Earth-mass planets in the Habitable Zone (HZ) around these low-mass stars, and (2) to uncover the distribution of planetary systems containing Earth-mass planets and more massive planets. To achieve these goals, we will carry out an extensive high-precision RV survey of $\sim 60$ carefully selected low-mass stars using 175 observing nights of the Subaru telescope over 5 years. Our initial target samples are collected from the literature and from the results of our pre-selection observations with optical spectroscopy of H-alpha lines. This will screen out active stars, leaving us with the targets best suited for very high precision RV measurements. Figure 12 shows our survey samples and comparison to the samples of other projects. According to a simulation of the RV observations based on both theoretical and empirical planet populations, our proposed observations can discover Earth-mass planets in close-in orbits with periods less than a few tens of days and super-Earths (5 Earth-masses) in orbits with periods less than a few hundred days. So far, only two Earth-mass planets in HZ have been reported with the RV method, and M-dwarf planetary companions with 1-100 Earth-masses beyond the snow line are known almost only from microlensing surveys. 


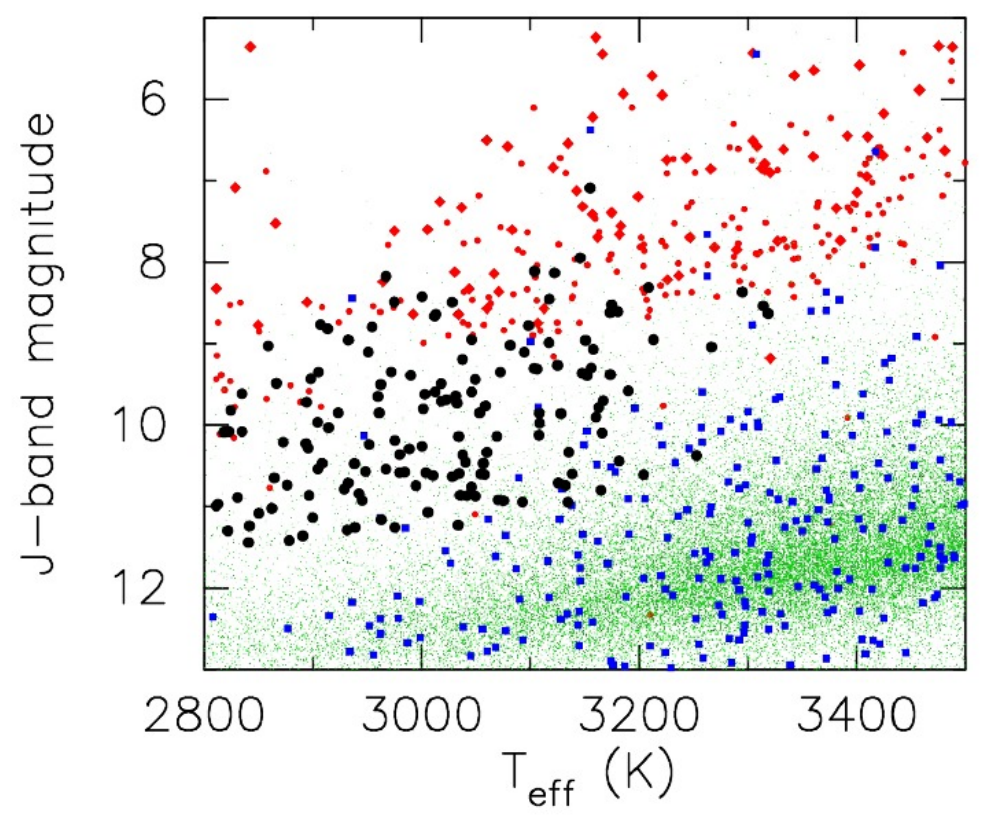

Figure 12. Teff-J mag distribution for M dwarfs in input catalogs of the past and currently planned exoplanet surveys. Sampled stars in the HARPS, IRD, CARMENES ${ }^{4}$, TESS surveys are plotted by red diamonds, black circles, red circles, and green dots (TIC6.2-CTL), respectively. The blue squares are the TESS planets expected based on Barclay et al ${ }^{5}$.

\section{CONCLUSION}

IRD is a near infrared, high resolution spectrometer for precise measurements of radial velocity of a nearby M-dwarfs. The special effort has been devoted to a stability of the instrument and many new technologies for example the ultra-low thermal expansion ceramic optics, the temperature control system, the laser frequency comb, etc. were developed. We demonstrated the instrumental stability is better than $2 \mathrm{~m} / \mathrm{s}$ from the laboratory experiments and some on-sky results. We have been continuing a commissioning of IRD at the Subaru telescope to characterize the instrument and the radial velocity stability on-sky.

\section{REFERENCES}

[1] Ishizuka, M., Kotani, T., Nishikawa, J., Kurokawa, T., Mori, T., Kokubo, T., Tamura, M., "Mode Scrambler for the Subaru Infrared Doppler Instrument (IRD)", PASP, 130, 988, 065003 (2018)

[2] T. Kokubo, T. Mori, T. Kurokawa, K. Kashiwagi, Y. Tanaka, T. Kotani, J. Nishikawa, and M. Tamura, "12.5GHz-spaced laser frequency comb covering Y, J, and H bands for Infrared Doppler instrument," Proc. SPIE 9912, 99121R (2016).

[3] Kuzuhara, M., et al. "Performance tests of Subaru/IRD for very precise and stable infrared radial velocity observations", this proceeding, Proc. SPIE (2018)

[4] Reiners, A., Zechmeister, M., Caballero, J. A., et al. 2017, ArXiv e-prints, arXiv:1711.06576

[5] Barclay, T., Pepper, J., \& Quintana, E. V. 2018, ArXiv e-prints, arXiv:1804.05050 